\title{
PREVALENCIA DE FACTORES DE RIESGO PARA SÎNDROME METABÓLICO Y SUS COMPONENTES EN USUARIOS DE COMEDORES POPULARES EN UN DISTRITO DE LIMA, PERÚ
}

\author{
Karen J. Adams 1,2,a, Jesús L. Chirinos ${ }^{3, b}$
}

\begin{abstract}
RESUMEN
Objetivos. Estimar la prevalencia de factores en riesgo para síndrome metabólico en adultos usuarios de los comedores populares del distrito de Lima. Materiales y métodos. Se realizó un estudio observacional, transversal, en usuarios de 20 a 59 años de comedores populares seleccionados de un muestreo bietápico por conglomerados. El síndrome metabólico se midió según la Federación Internacional de Diabetes (FID). Se evaluaron factores de riesgo propuestos por la OMS y la FID, así como las necesidades e ingesta promedio de la dieta y sus componentes en el almuerzo. Resultados. La prevalencia global de síndrome metabólico fue de $40,1 \%$, las mujeres presentaron una prevalencia de $30,4 \%$ y los varones $24 \%$, la prevalencia de los componentes del síndrome metabólico fueron obesidad abdominal 51,6\%, C-HDL bajo $42,2 \%$, hipertrigliceridemia $35,3 \%$, obesidad $43,3 \%$, sobrepeso $35,8 \%$, hipertensión arterial $21,0 \%$ e hiperglicemia $14,1 \%$. El sedentarismo se presenta en el $86,1 \%$ de los participantes. Conclusiones. El sobrepeso, la obesidad abdominal y colesterol alto, fueron los factores de riesgo con mayor prevalencia en mujeres. El componente de síndrome metabólico de mayor prevalencia en los usuarios de comedores populares del distrito de Lima es la obesidad abdominal.
\end{abstract}

Palabras clave: Síndrome metabólico; Adultos; Factores de riesgo; Comedores populares (Fuente: DeCS BIREME).

\section{PREVALENCE OF RISK FACTORS FOR METABOLIC SYNDROME AND ITS COMPONENTS IN COMMUNITY KITCHEN USERS IN A DISTRICT IN LIMA, PERU}

\begin{abstract}
Objectives. To estimate the prevalence of risk factors for metabolic syndrome in adults who are community kitchen-users in a district in Lima. Materials and Methods. An observational, cross-sectional study was carried out with 20 to 59 -year-old users of community kitchens selected from a two-stage cluster sampling. The metabolic syndrome was measured according to the International Diabetes Federation (IDF). Risk factors proposed by the WHO and the IDF were assessed, as well as the diet needs and intake average and its components at lunch. Results. The global prevalence of metabolic syndrome was $40.1 \%$ : women exhibited a $30.4 \%$ prevalence and men $24 \%$. Prevalence of the metabolic syndrome components were, abdominal obesity $51.6 \%$, low C-HDL $42.2 \%$, hypertriglyceridemia $35.3 \%$, obesity $43.3 \%$, overweight $35.8 \%$, arterial hypertension $21.0 \%$, and hyperglycemia $14.1 \%$. A sedentary lifestyle is present in $86.1 \%$ of participants. Conclusions. Excess weight, abdominal obesity, and high cholesterol were the most prevalent risk factors in women. The metabolic syndrome component with the highest prevalence in community kitchen users in a district of Lima is abdominal obesity.
\end{abstract}

Keywords: Metabolic syndrome; Adults; Risk factor's; Popular social dinning (Source: DeCS BIREME)

\section{INTRODUCCIÓN}

Las enfermedades crónicas no transmisibles (ECNT) presentan una alta prevalencia, sobre todo en países en desarrollo, impactando en los costos sanitarios, así como en la morbilidad, mortalidad y la productividad de las personas ${ }^{(1)}$.

La prevalencia de síndrome metabólico (SM) utilizando los criterios de ATP III (2) en el Perú, fluctúa entre el 10 y $45 \%$. En la Encuesta de Indicadores Nacionales para
ECNT ${ }^{(3)}$ usando los criterios de la Federación Internacional de Diabetes (FID), se encontró una prevalencia de SM de $16,6 \%$ en varones y $34,3 \%$ en mujeres, siendo mayor en población de la ciudad de Lima $(28,8 \%)$ en comparación con otras regiones. La frecuencia de los componentes del SM fue: obesidad abdominal en $65,6 \%$, colesterol-HDL bajo en $54,2 \%$, hipertrigliceridemia en $30 \%$, hipertensión arterial en $19,1 \%$ e hiperglicemia en $8 \%{ }^{3}$.

La nueva definición planteada por la FID ${ }^{(4)}$ considera a los grupos étnicos para el diagnóstico de obesidad central. Esto

\footnotetext{
Departamento Académico de Ciencias de la Salud, Universidad San Ignacio de Loyola. Lima, Perú.

Ciencias de la Salud, Universidad Científica del Sur. Lima, Perú.

Facultad de Salud Pública y Administración, Universidad Peruana Cayetano Heredia. Lima, Perú.

a Doctora en Nutrición; ${ }^{\text {b }}$ Médico cirujano Doctor en Salud Pública.

Recibido: 26/06/2017 Aprobado: 29/12/2017 En línea: 05/04/2018
}

Citar como: Adams KJ, Chirinos JL. Prevalencia de factores de riesgo para síndrome metabólico y sus componentes en usuarios de comedores populares en un distrito de Lima, Perú. Rev Peru Med Exp Salud Publica. 2018;35(1):39-45. 10.17843/rpmesp.2018.351.3598. 
permite obtener información confiable sobre la magnitud del SM en estudios epidemiológicos, independientemente del país. Según la FID (4), una persona padece de SM, si además del perímetro de cintura elevado según etnia (obesidad abdominal) presenta dos de los siguientes signos: elevación de triglicéridos plasmáticos, bajas concentraciones plasmáticas de C-HDL, hipertensión arterial e hiperglicemias en ayunas. A diferencia de otras definiciones de SM, estos criterios consideran a la circunferencia de cintura como un factor fundamental para el SM.

En el Perú, los comedores populares (CP) surgieron a finales de los años 70 con las migraciones a la ciudad ${ }^{(5)}$. En la actualidad los CP son regidos por el Ministerio de Desarrollo e Inclusión Social (MIDIS) en el marco de ejecución de los programas sociales, uno de ellos es el Programa de Complementación Alimentaria (PCA) ${ }^{(6)}$. Debido al proceso de descentralización, los CP están a cargo de los municipios provinciales o distritales ${ }^{(6)}$. En dichos espacios encontramos a mujeres organizadas, amas de casa, vecinas de barrio que se reúnen para brindar raciones alimenticias a familias pobres y en extrema pobreza ${ }^{(7)}$.

Las raciones alimenticias tienen bajo costo gracias a las donaciones o subsidios, la adquisición de productos por cantidad y el número de alimentos preparados. Los ingresos son obtenidos del precio que pagan los comensales (en promedio 0,62 dólares americanos) que hacen uso del comedor durante los 26 días al mes que funcionan. Los componentes nutricionales de esta ración deben cubrir el $31 \%$ de los requerimientos de calorías diarias y el $54 \%$ de proteínas de una persona ${ }^{(6)}$.

Los beneficiarios de dichos CP se encuentran expuestos a cambios en la dieta y factores ambientales, los cuales incrementan el riesgo de obesidad, diabetes, dislipemia, hipertensión, todas asociadas al SM ${ }^{(8)}$. El presente estudio propone identificar las características asociadas al SM en una muestra seleccionada de los beneficiarios adultos de los comedores populares del distrito de Lima, Perú.

\section{MATERIALES Y MÉTODOS}

\section{DISEÑO Y POBLACIÓN DE ESTUDIO}

Se realizó un estudio transversal y analítico entre los meses de enero a marzo de 2015. Se incluyó personas de ambos sexos entre los 20 y 59 años beneficiarios de los CP en el distrito de Lima. Se excluyeron personas con enfermedad crónica terminal, alguna deficiencia mental o dificultad de expresión, enfermedad consuntiva (neoplasia, tuberculosis), gestantes o con alguna enfermedad aguda durante el estudio.

El universo de la población fue de 10185 beneficiarios agrupados en $83 \mathrm{CP}$, siendo estos conglomerados

\section{MENSAJES CLAVE}

Motivación para realizar el estudio: En el Perú los comedores populares brindan raciones alimenticias a familias pobres y en extrema pobreza estas raciones alimenticias tienen bajo costo pero que deben cubrir los requerimientos de calorías y de proteínas diarias de una persona.

Principales hallazgos: Cuatro de diez beneficiarios de comedores en populares en Lima tienen síndrome metabólico, estando además dos terceras partes de los beneficiarios con sobrepeso y obesidad.

Implicancias: El sobrepeso y la obesidad son frecuentes lo cual se podría explicar por los estilos de vida y alteraciones en los patrones de alimentación. Estos datos son preocupantes debido al incrementa en el riesgo de mortalidad prematura generado por las enfermedades cardiovasculares.

naturales. Se realizó un muestreo por conglomerados, bietápico, estratificado y seleccionado por cuotas. En la primera etapa se seleccionó una muestra de $\mathrm{CP}$ en forma aleatoria y con probabilidades proporcionales al tamaño de cada conglomerado. En la segunda etapa, se eligió una muestra de beneficiarios en los conglomerados seleccionados. Dicho sub muestreo fue estratificado por sexo y se asignaron cuotas proporcionales a cada rango de edad (grupo quinquenal).

Para el tamaño de la muestra se utilizó la fórmula de muestreo aleatorio simple para poblaciones finitas, considerando un nivel de confianza de $95 \%$, error absoluto de $6 \%$ y prevalencia de obesidad abdominal $65,6 \%$, según lo reportado por Cárdenas (2009) ${ }^{(3)}$.

Para la selección muestral, en primera instancia fueron seleccionados aleatoriamente ocho conglomerados, posteriormente, elaboramos un listado de beneficiarios agrupados por pertenencia a CP. Los beneficiarios fueron seleccionados mediante un muestreo sistemático con arranque aleatorio, permitiendo obtener beneficiarios de los ocho CP. El tamaño de muestra por CP fue determinado por afijación proporcional a la raíz cuadrada del tamaño del conglomerado. Además, se asignó proporciones por CP según sexo y grupos quinquenales de edad, según estimaciones del Instituto Nacional de Estadística e Informática (INEI) ${ }^{(9,12)}$ para Lima Metropolitana. Los conglomerados tuvieron un total de 2137 beneficiarios, el tamaño muestral final fue de 374 .

\section{MEDICIÓN DE VARIABLES}

La determinación de SM fue realizada usando los criterios de la Federación Internacional de Diabetes ${ }^{(4)}$. El perímetro abdominal fue medido con cinta métrica antropométrica marca SECA, con capacidad de $200 \mathrm{~cm}$ y precisión de 1 
$\mathrm{mm}$, la medición fue hecha a nivel del punto medio entre el borde inferior de las costillas y la cresta iliaca ${ }^{(10)}$. Para determinar obesidad abdominal se usó los valores de referencia según grupo étnico propuesto por la FID ${ }^{(4)}$. Se consideró que tenían obesidad abdominal los varones con circunferencia abdominal $\geq$ de $90 \mathrm{~cm}$ y las mujeres que tuvieran $\geq$ de $80 \mathrm{~cm}$.

Dislipidemia y glicemia fue medida en muestra sanguínea tomada en ayunas. La determinación de glucosa, colesterol total (CT) y triglicéridos (TG) se obtuvo por métodos enzimáticos colorimétricos según Trinder. El colesterol-HDL (C-HDL) se determinó previa precipitación de lipoproteína de baja (LDL) y muy baja densidad y (VLDL), empleando el sistema enzimático colesterol oxidasa/peroxidasa con colorimetría según Trinder. Las muestras fueron procesadas en autoanalizadores con calibradores y sueros controles.

Se consideró hipertrigliceridemia a los valores $\geq 150 \mathrm{mg} / \mathrm{dL}$ con independencia de género. Se consideró con C-HDL bajo a quienes tuvieran valores $<40 \mathrm{mg} / \mathrm{dL}$ en varones y $<50 \mathrm{mg} / \mathrm{dL}$ en mujeres. Se consideró glicemias alteradas en ayunas a concentraciones de glucosa $\geq 100 \mathrm{mg} / \mathrm{dL}^{(4)}$. No se tuvo en cuenta la medicación previa de los sujetos.

La medición de tensión arterial fue realizada con tensiómetro Mac-Check-501. Se consideró hipertensión arterial cuando la presión sistólica era $\geq 130 \mathrm{~mm}$ de $\mathrm{Hg}$ o diastólica $\geq 85$ $\mathrm{mm}$ de $\mathrm{Hg}^{(4)}$. No se tuvo en cuenta la medicación previa de los sujetos.

La evaluación del estado nutricional se realizó según los estándares de la Organización Mundial de Salud (OMS) ${ }^{(10)}$ para jóvenes entre 20 a 29 años y adultos entre 30 a 59 años utilizando el índice de masa corporal (IMC). Se clasificó como delgadez I: IMC <16; delgadez II: IMC $\geq 16$ y $\leq 16,9$; delgadez II: IMC $\geq 17$ y $\leq 18,4$; normal: IMC $\geq 18,5$ y $\leq$ 24,9 ; sobrepeso: IMC $\geq 25$ y $\leq 29,9$; obesidad I: IMC $\geq 30$ y $\leq 34,9$; obesidad II: IMC $\geq 35$ y $\leq 39,9 ;$ y obesidad III: IMC $\geq 40$.

La actividad física se midió con cuestionario siguiendo las recomendaciones sobre Actividad Física para la Salud (11) de la OMS en adultos de 18 a 64 años. El consumo de alimentos, fue medido a través del consumo individual utilizando el recordatorio de 24 horas, la pesada directa y la frecuencia de consumo de alimentos ${ }^{(12)}$ :

a) En el recordatorio de 24 horas ${ }^{(12)}$, se solicitó al participante que recuerde todos los alimentos y bebidas ingeridos en el día anterior, fueron mostradas láminas con alimentos de diversos tamaños y/o medidas caseras para ayudar al entrevistado a cuantificar las cantidades físicas de alimentos y bebidas consumidos. (Guía visual de las dimensiones de las unidades de consumo. Tablas auxiliares para la formulación de regímenes alimentarios. INS. 2014) ${ }^{(13)}$. b) Para la pesada directa de los alimentos (12), durante ocho días en los ocho CP se realizó la técnica de pesado directo de los alimentos en el almuerzo (segundo, sopa y refresco). Para que la cantidad de ingredientes preparados y consumidos sea lo más exacto posible, se utilizó una balanza marca Soehnle con aproximación de $10 \mathrm{~g}$, un juego de medidas de capacidad de 1/4, 1/2 y 1 litro para tomar medidas de volúmenes de líquidos o agua, y un centímetro para medir el diámetro de los recipientes utilizados para la preparación de los alimentos y para medir los volúmenes pequeños una jeringa descartable de 20 cc. Solo se consideraron los macronutrientes (calorías, carbohidratos, proteínas, grasas).

c) Frecuencia de consumo de alimentos ${ }^{(12)}$, se utilizó una lista cerrada de alimentos sobre la que se solicita la frecuencia (diaria, semanal o mensual) de consumo de cada uno de ellos.

\section{TÉCNICAS Y PROCEDIMIENTOS DE RECOLECCIÓN DE DATOS}

La subgerencia de Programas Alimentarios de la Municipalidad Metropolitana de Lima proporcionó la información y datos de los CP. Luego de los procedimientos de selección, se visitaron los ocho CP para explicar la investigación a las representantes, quienes brindaron su autorización. En una segunda visita se explicó a los usuarios la finalidad de la investigación.

En una tercera visita en cada $\mathrm{CP}$, se procedió al llenado de una ficha de recolección de datos incluyendo hábitos nocivos, consumo de alimentos (recordatorio de 24 horas) y actividad física, mediante una entrevista. Seguidamente a los participantes se les realizó una evaluación antropométrica (peso y talla), la medición del perímetro abdominal, se tomó el control de presión arterial, y la toma de muestra para los análisis de laboratorio.

Se realizó el control de calidad de los materiales antropométricos (balanza y tallímetro) aplicando una lista de cotejo, y las técnicas de medición del peso, talla y perímetro abdominal en cada CP y al momento de la evaluación. Se realizaron pruebas de estandarización con el equipo de campo para evaluar la precisión y la exactitud de los antropometristas.

Finalmente, se visitaron los ocho CP por ocho días para realizar la pesada directa de la ración del almuerzo. Para estimar los requerimientos de energía de cada usuario se cálculo dicho requerimiento para jóvenes y adultos, residentes en área urbana y según sexo ${ }^{(14)}$, también se realizó el cálculo de las proteínas ${ }^{(15)}$. De acuerdo con la información del MIDIS ${ }^{(6)}$, los componentes nutricionales de 
la ración ofrecida deben cubrir el $31 \%$ de los requerimientos de calorías y el $54 \%$ de proteínas de una persona. Se realizó la distribución porcentual ${ }^{(16-18)}$ de energía y macronutrientes para hallar el requerimiento solo en el almuerzo y fue comparada la ingesta en los CP.

\section{ANÁLISIS DE DATOS}

Para determinar si los datos se ajustan o no a una distribución normal se realizó la prueba de Kolmogorov-Smirnov. Para variables que no seguían una distribución normal se utilizó la prueba $U$ de Mann-Whitney. El análisis estadístico fue realizado en el programa estadístico SPSS versión 20 .

\section{CONSIDERACIONES ÉTICAS}

Todos los participantes fueron informados de los objetivos del estudio, los procedimientos se aplicaron previo consentimiento informado por escrito. El estudio fue aprobado por el Comité de Ética de la Universidad Peruana Cayetano Heredia.

\section{RESULTADOS}

Se estudiaron 374 beneficiarios de CP, de los cuales el $71,1 \%$ fueron mujeres. El $46 \%$ tuvo entre 20 y 39 años y el $54 \%$ entre 40 y 59 años. La prevalencia global de SM fue de 40,1\% (IC95\%: 35,1-45,1), en mujeres la prevalencia fue de $42,9 \%(114 / 266)$, mientras que en varones afectó al $33,3 \%(36 / 108)$, dicha diferencia fue estadísticamente significativa $(p<0,01)$. El componente más frecuente es la obesidad abdominal, especialmente en mujeres con $57,5 \%$ (IC95\%: 51,6-63,5), mientras que en varones afectó al $37,0 \%$ (IC95\%: 27,9-46,1). El segundo componente más frecuente fue el C-HDL bajo, llegando en mujeres a 50,8\% (IC95\%: 44,7-56,8) (Figura 1).

Las prevalencias de factores de riesgo según sexo se presentan en la Tabla 1, siendo mayor para las mujeres el sobrepeso con $34,2 \%$. El sedentarismo se presenta en el $86,1 \%$ de los participantes, siendo para mujeres $86,8 \%$ (IC95\%: 82,8-90,0), y en varones 84,3\% (IC95\%: 77,4$91,1)$.

De acuerdo con la evaluación del consumo de alimentos la ingesta energética por ración de almuerzo fue 731,87 $\pm 44,58 \mathrm{kcal} / \mathrm{día}$, sin embargo, la necesidad calórica promedio es de $555,16 \pm 97,90 \mathrm{kcal} /$ día. Por otro lado, el requerimiento de proteínas $(100,23 \pm 20,23 \mathrm{kcal} / \mathrm{día})$ no es cubierto por la ingesta promedio de proteínas en los CP $(78,71+3,06 \mathrm{kcal} / \mathrm{día})$ (Tabla 2).

Se obtuvo un porcentaje de adecuación por encima de las necesidades nutricionales en un $31,8 \%$ para las calorías totales en el almuerzo. En las proteínas, se observa un porcentaje de adecuación por debajo en un 21,5\%. La proporción de calorías provenientes de la grasa se encuentra por encima de los valores recomendados en un $25,1 \%$, mientras que para las calorías provenientes del consumo de carbohidratos los valores se encuentran $57 \%$ por encima de la recomendación nutricional (Figura 2).

\section{DISCUSIÓN}

El estudio muestra que cuatro de diez beneficiarios de CP del distrito de Lima tienen SM, destaca además que el componente de SM más prevalente es la obesidad abdominal. Por otro lado, dos terceras partes de los beneficiarios tienen sobrepeso y obesidad. Estos datos son preocupantes debido al incremento en el riesgo de mortalidad prematura generado por las enfermedades cardiovasculares. El sobrepeso y la obesidad son frecuentes en Lima Metropolitana y la costa de Perú (19), lo cual se podría explicar por la mayor urbanización y desarrollo económico que genera cambios en los estilos de vida, y

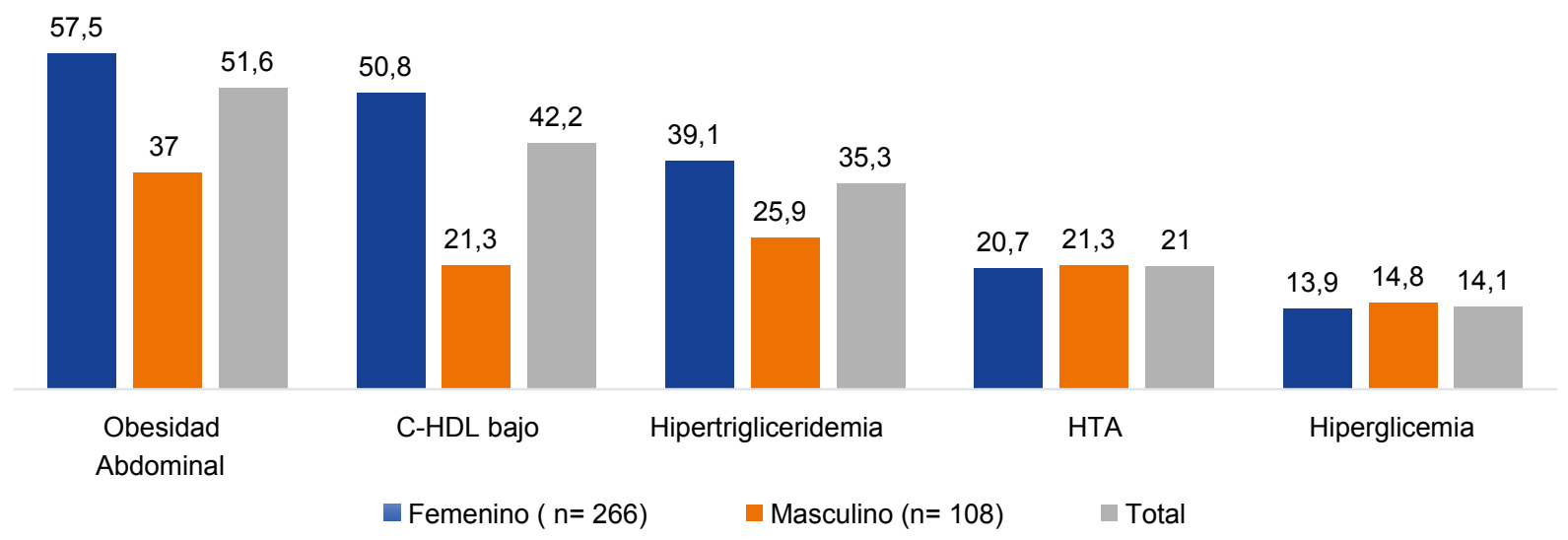

Figura 1. Prevalencia de componentes del síndrome metabólico en usuarios de comedores populares de un distrito de Lima 
Tabla 1. Frecuencia de factores de riesgo para síndrome metabólico por sexo en usuarios de comedores populares del distrito de Lima, Perú

\begin{tabular}{|c|c|c|c|c|c|c|}
\hline & \multicolumn{3}{|c|}{ Femenino $(n=266)$} & \multicolumn{3}{|c|}{ Masculino $(n=108)$} \\
\hline & $\mathbf{n}$ & $\%$ & IC $95 \%$ & $\mathbf{n}$ & $\%$ & IC $95 \%$ \\
\hline \multicolumn{7}{|c|}{ Estado nutricional } \\
\hline Normal & 50 & 18,8 & $14,1-23,5$ & 28 & 10,5 & $4,7-16,3$ \\
\hline Sobrepeso & 91 & 34,2 & $28,5-39,9$ & 43 & 16,2 & $9,2-23,1$ \\
\hline Obesidad I & 80 & 30,1 & $24,6-35,6$ & 25 & 9,4 & $3,9-14,9$ \\
\hline Obesidad II & 28 & 10,5 & $6,8-14,2$ & 7 & 2,6 & $0,0-5,7$ \\
\hline \multicolumn{7}{|c|}{ Consumo de alcohol } \\
\hline Sí & 86 & 32,3 & $26,7-37,9$ & 33 & 30,6 & $21,9-39,2$ \\
\hline No & 180 & 67,7 & $62,0-73,3$ & 75 & 69,4 & $60,8-78,1$ \\
\hline \multicolumn{7}{|c|}{ Consumo de tabaco } \\
\hline Sí & 45 & 16,9 & $12,4-21,4$ & 21 & 19,4 & $12,0-26,9$ \\
\hline No & 221 & 83,1 & $78,6-87,6$ & 87 & 80,6 & $73,1-88,0$ \\
\hline \multicolumn{7}{|l|}{ Actividad física } \\
\hline Sí & 35 & 13,2 & $9,1-17,2$ & 17 & 15,7 & $8,9-22,6$ \\
\hline No & 231 & 86,8 & $82,8-90,9$ & 91 & 84,3 & $77,4-91,1$ \\
\hline
\end{tabular}

provocan alteraciones en los patrones de alimentación y la actividad física.

El sobrepeso y obesidad son factores de riesgo para el desarrollo de enfermedades cardiovasculares no transmisibles, diabetes tipo 2, hipertensión arterial, dislipidemia, cáncer y apnea del sueño; estos factores son altamente prevalentes en la población usuaria de los comedores populares con mayor predominio en las mujeres. Álvarez et al. ${ }^{(19)}$, quién calculó el exceso de peso a nivel nacional, también encontró una alta prevalencia en mujeres adultas con el $65,8 \%$. Otro estudio donde participaron mujeres de comedores populares de un distrito de Lima Metropolitana, muestra prevalencias más elevadas con obesidad abdominal por encima del $90 \%$, y sobrepeso/ obesidad valores superiores al $73 \%{ }^{(20)}$.

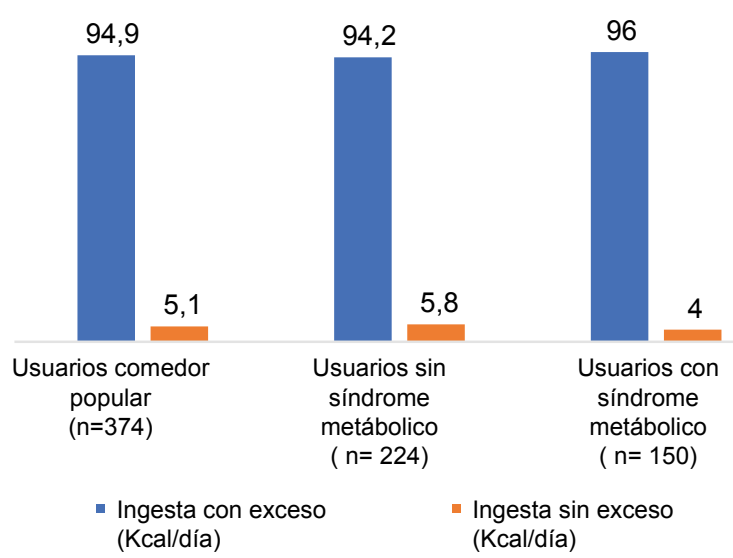

Figura 2. Ingesta de calorías consumidas según condición respecto al síndrome metabólico en los usuarios de comedores populares del distrito de Lima, Perú.
El componente más frecuente del SM fue la obesidad abdominal, los resultados muestran que uno de cada dos beneficiarios lo presentan, siendo más afectadas las mujeres. Un estudio desarrollado en usuarias de comedores populares en los distritos de Lima, Callao, La Libertad y Arequipa reportó resultados similares de obesidad abdominal $(51,9 \%)^{(8)}$. Nuestro estudio muestra además, que uno de cada cuatro usuarios de comedores populares presenta hipertensión, resultados similares a los encontrados en mujeres de comedores populares de Lima y Callao ${ }^{(8)}$.

El sedentarismo, se observa en uno de cada dos personas en los comedores populares. Es fundamental incrementar la actividad física conduciendo a disminuir los factores de riesgo para síndrome metabólico ${ }^{(17)}$.

Respecto a la respuesta brindada por los participantes frente al consumo de alcohol y tabaco, diversas investigaciones en Perú revelan que los varones realizan esta práctica con mayor frecuencia; sin embargo, lo encontrado en esta población muestra lo contrario, probablemente debido a la proporción de participantes mujeres en nuestro estudio en $\mathrm{CP}$.

Respecto al patrón de consumo este estudio encontró que en la ración servida al usuario del comedor popular hay un predominio de carbohidratos y una escasa presencia de fibra dietaria. La proporción de calorías provenientes de la grasa y de carbohidratos se encuentra por encima de la adecuación a las recomendaciones nutricionales ${ }^{(14)}$.

Por lo tanto, los CP exceden los requerimientos en energía y hay un déficit en el aporte proteico. Los hallazgos en este escenario puntual, brindan evidencias para focalizar 
Tabla 2. Necesidades e ingesta promedio de la dieta y sus componentes en los usuarios de los comedores populares

\begin{tabular}{|c|c|c|}
\hline & $\begin{array}{c}\text { Necesidades } \\
\text { Ración de comida (almuerzo) }\end{array}$ & $\begin{array}{c}\text { Ingesta } \\
\text { Ración de comida (almuerzo) }\end{array}$ \\
\hline & Media \pm DE & Media \pm DE \\
\hline \multicolumn{3}{|l|}{ Nutrientes } \\
\hline Dieta (kcal/día) ${ }^{a}$ & $555,16 \pm 97,90$ & $731,87 \pm 44,58$ \\
\hline Proteínas (kcal/día) ${ }^{b}$ & $100,23 \pm 20,23$ & $78,71 \pm 3,06$ \\
\hline Grasa (kcal/día) ${ }^{c}$ & $138,79 \pm 24,47$ & $173,77 \pm 32,44$ \\
\hline Carbohidratos (kcal/día) ${ }^{d}$ & $305,34+53,84$ & $479,39+60,49$ \\
\hline
\end{tabular}

${ }^{a}$ Cálculo realizado según necesidades energéticas para la Población Peruana. MINSA 2012. ${ }^{(14)}$

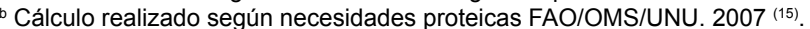

c,d Distribución Porcentual de Macronutrientes $(19,20,21,22)$

intervenciones en los CP dirigidas a reducir la ocurrencia del SM, así como de sus componentes.

Como limitaciones del estudio encontramos el diseño transversal que no permite establecer una relación de causalidad entre los factores asociados y el síndrome metabólico, sin embargo, dicha asociación es consistente con lo reportado en otros estudios. Si bien, la selección de participantes fue probabilística, el diseño muestral solo es representativo para las poblaciones estudiadas, y no pretenden ser estimaciones para la población de mujeres y varones adultos en la ciudad de Lima.
En conclusión, el componente de síndrome metabólico de mayor prevalencia en los usuarios de comedores populares del distrito de Lima es la obesidad abdominal.

Contribuciones de los autores: KAU ha participado en la concepción del artículo, la recolección de datos, su redacción, financiar la investigación y aprobación de la versión final. Además, KAU realizó el análisis de datos. JLCH se encargó de la aprobación final y revisión crítica del artículo.

Fuentes de financiamiento: autofinanciado

Conflictos de interés: Los autores declaran no tener conflictos de interés

\section{REFERENCIAS BIBLIOGRÁFICAS}

1. Villena JE. Diabetes mellitus in Peru. Ann Glob Health. 2015;81(6):765-775. doi: 10.1016/j.aogh.2015.12.018.

2. National Cholesterol Education Program (NCEP) Expert Panel on Detection, Evaluation, and Treatment of High Blood Cholesterol in Adults (Adult Treatment Panel III). Third report of the National Cholesterol Education Program (NCEP) Expert Panel on Detection, Evaluation, and Treatment of High Blood Cholesterol in Adults (Adult Treatment Panel III) final report. Circulation. 2002;106(25):3143-421.

3. Cárdenas $\mathrm{H}$, Sánchez J, Roldán L, Mendoza F. Prevalencia del síndrome metabólico en personas a partir de 20 años: Perú, 2005. Rev Esp Salud Publica. 2009;83(2): 257-265.

4. International Diabetes Federation (IDF) [Internet]. The IDF consensus worldwide definition of the metabolic syndrome; 2006 [Citado el 01 de noviembre del 2017] Disponible en: http://www.idf.
org/webdata/docs/IDF_Meta_def_ final.pdf.

5. Garret J. Comedores Populares: Lessons for Urban Programming from Peruvian Community Kitchens (IFPRI, Washington, D.C., 2001). Disponible en: http:// www.ncbi.nlm.nih.gov/pubmedhealth/ PMH0009180/

6. Ministerio de Desarrollo e Inclusión Social. [Internet]. Lima. Perú. 2017. [actualizado 4 Nov 2017; citado 4 abr 2016]. Disponible en: www.midis.gob.pe/

7. Blondet C, Trivelli C. Cucharas en alto. Del asistencialismo al desarrollo local: fortaleciendo la participación de las mujeres. Lima: Instituto de Estudios Peruanos; 2004

8. Núñez-Robles E, Huapaya-Pizarro C, Torres-Lao R, Esquivel-León S, Suarez-Moreno $\mathrm{V}$, Yasuda-Espinoza $\mathrm{M}$, et al. Prevalencia de factores de riesgo cardiovascular y riesgo metabólico en escolares, universitarios y mujeres de organizaciones sociales de base en distritos de Lima,
Callao, La Libertad y Arequipa, Perú 2011. Rev Peru Med Exp Salud Publica. 2014;31(4):652-659.

9. Instituto Nacional de Estadística; Perú. Estimaciones y Proyecciones de población por departamento, sexo y grupos quinquenales de edad 1995-2025. Lima: INEI; 2009.

10. Organización Mundial de la Salud. Obesity: Preventing and managing the global epidemic. WHO Technical report series. Geneva (Switzerland):OMS; 2000.

11. Organización Mundial de la Salud. Recomendaciones mundiales sobre actividad física para la salud. Ginebra (Suiza): Organización Mundial de la Salud; 2010.

12. Instituto Nacional de Salud. Vigilancia de indicadores nutricionales: Módulo de capacitación. Lima (Perú): Ministerio de Salud; 2013.

13. Instituto Nacional de Salud. Tablas auxiliares para la formulación y evaluación de regímenes alimentarios. Lima (Perú): Ministerio de Salud; 2014. 
14. Instituto Nacional de Salud. Requerimientos de energía para la población peruana. Lima (Perú): Ministerio de Salud; 2012.

15. Joint WHO/FAO/UNU Expert Consultation. Protein and amino acid requirements in human nutrition. World Health Organ Tech Rep Ser. 2007;(935):1-265.

16. Franz MJ, Bantle JP, Beebe CA, Brunzell JD, Chiasson JL, Garg A, et al. Evidence-based nutrition principles and recommendations for the treatment and prevention of diabetes and related complications. Diabetes Care 2003; 26(1):S51-S61.
17. American Diabetes Association: Nutrition recommendations and interventions for diabetes. Diabetes Care 2007; 30(1):S48S65. Doi: $10.2337 / \mathrm{dc} 07-S 048$

18. Evert AB, BoucherJL, Cypress M, Dunbar SA, Franz MJ, Mayer-Davis EJ, et al. Nutrition therapy recommendations for the management of adults with diabetes. Diabetes Care, 2013 Nov;36(11):382142. doi: $10.2337 / \mathrm{dc} 13-2042$.

19. Álvarez-Dongo D, Sánchez-Abanto J, Gómez-Guizado G, Tarqui-Mamani C. Sobrepeso y obesidad: prevalencia y determinantes sociales del exceso de peso en la población peruana (2009-
2010). Rev Peru Med Exp Salud Pública. 2012;29(3):303-13.

20. Hidalgo MAJ, Chuquinaupa AI L, Luna JMF. Factores de riesgo de Síndrome Metabólicoen mujeres sociasdeComedores Populares del Cercado de Lima. Rev Peru Cardiologia, (2009):35(2):91.

Correspondencia: Karen Adams Ubaldo

Dirección: Calle Tnte Fap César Concha 110 - Dpto. 502. Urb. Liguria. Santiago de Surco. Lima, Perú

Teléfono: 966367347

Correo electrónico:adams_nutrition@hotmail.com

\section{¿CÓMO ENVIAR UN ARTÍCULO A NUESTIRA REVISTA?}

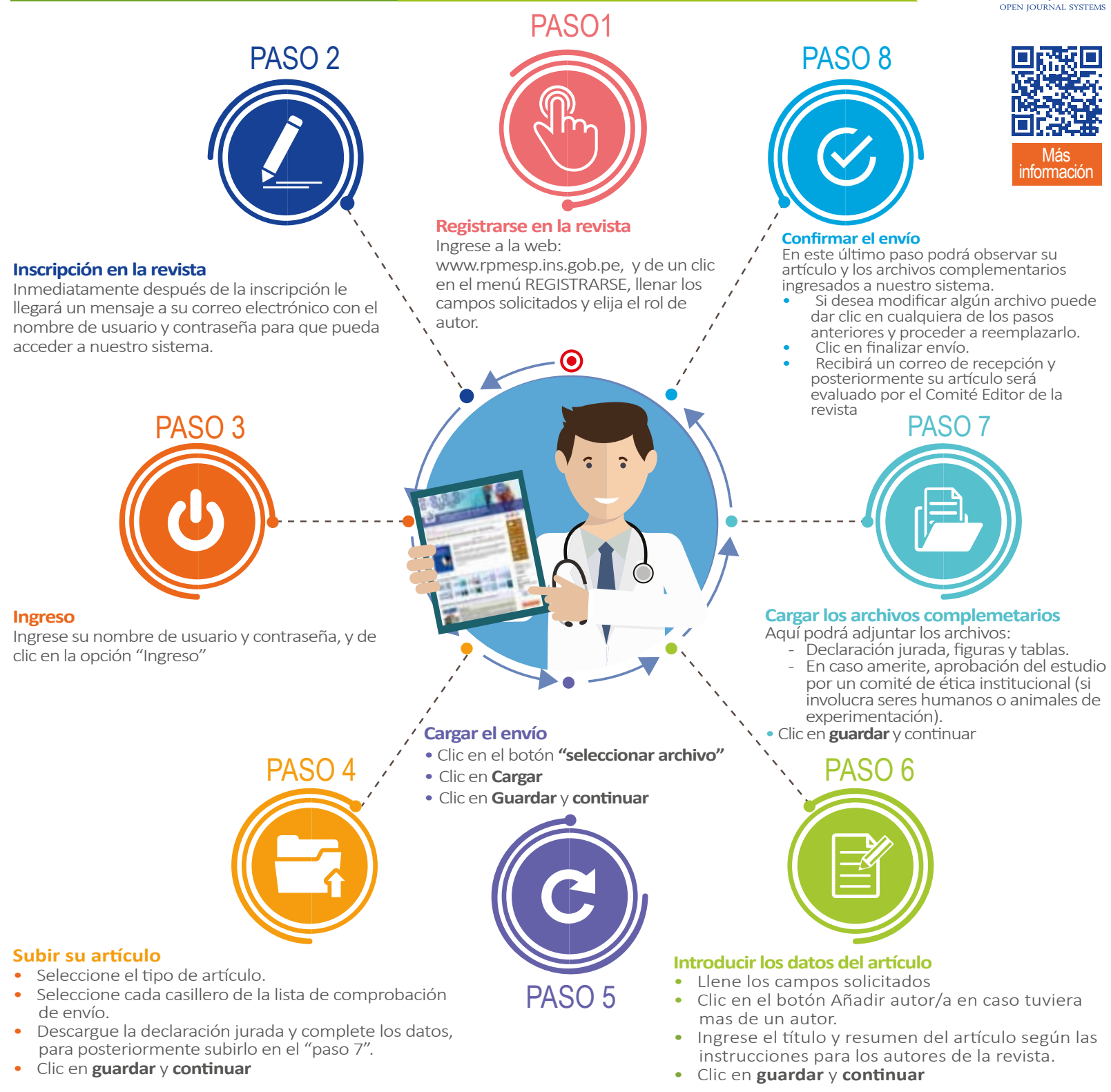

